\title{
The Research on Industrial Information Monitoring System Based on B/S Structure
}

\author{
Xuexuan $\mathrm{ZHU}^{1, \text { a }}$ \\ ${ }^{1}$ College of Electrical and Electronic Engineering, Xin Yu University, Xin Yu 338004, China \\ axuexuanzhu@126.com
}

Keywords: Industrial Information Monitoring; Process Monitoring; B/S Structure

\begin{abstract}
With the rapid development of network technology and the advance of information technology companies, the traditional monitoring system and web technology combined with the $\mathrm{B} / \mathrm{S}$ mode of the computer remote monitoring system has gradually become a new research and development of hot spots, built on B/S's industrial monitoring system has become one of the development direction of industrial control field. In this paper, the real-time communication technology to achieve industrial control data sharing between the network and the enterprise information network, was designed based on real-time remote monitoring system structure B/S technology, we can see that this design based on B/S surveillance systems to better meet the user's requirements, to achieve the industrial production process monitoring.
\end{abstract}

\section{Introduction}

With the continuous development of network technology, the technology has penetrated into all areas of daily life and industrial production, which makes the operation of the remote monitoring of equipment possible. Through remote real-time monitoring, technical personnel are not required to visit the site, especially in the harsh environment can run on the scene of a variety of devices to be monitored. Remote monitoring technology is a combination of network technology, computer technology and control technology, which leverages the existing LAN and WAN infrastructure for the plant internal LAN monitoring and regional monitoring provides an effective control method [1-3]. This article is based on the needs of enterprise management and market applications, and B/S mode is introduced into the monitoring system, selected based on B/S mode information monitoring system for industrial research and implementation.

Industrial Information Monitoring System B/S mode as the monitoring system is based on a communication platform technology-based technology, which is simple, efficient, cross-platform, etc., it has become an information network of the most widely used platform for information exchange , the use of the browser as a unified client, as opposed to the traditional client-server model, B/S mode consistent user interface, friendly and convenient for users to use the software installation, maintenance and upgrades easy, as long as the client has a browser you can access the system with good scalability, extensions work only done on the server, all the client software does not need to change, the lack of these features is the mode, the technology greatly reduces the cost of the system, to facilitate the users [4]. Significance of B/S mode, real-time monitoring of industrial information technology for the future development trend of industrial real-time monitoring information done a very good attempt.

\section{Architecture of B/S Mode}

Because of these deficiencies $\mathrm{C} / \mathrm{S}$ structure, we can add in the middle of the traditional $\mathrm{C} / \mathrm{S}$ structure layer, the client is responsible for the original function to the intermediate layer to achieve, the middle layer is the server layer. Thus, the client is not responsible for the original data access, we only need to install the client browser on it. The original server as the database server, install the database management system and create a database on the database server. The role of the database server is accessed, and passed to the browser through the work network. Thus, both the server browser server, and database server browser [5-6]. In this mode, the client becomes a simple 
browser, forming a B/S mode, this is what we studied browser server mode. Based on the structure of $\mathrm{C} / \mathrm{S}$ mode it will be combined with a database, form-based computing model of the database and the application of the model to the middle, forming a three-tier client-server application structure, the three-tier structure of the application system of three functions levels were clearly divided [7], it is logically independent of its architecture shown in Figure 1.

Browser Web server Database server

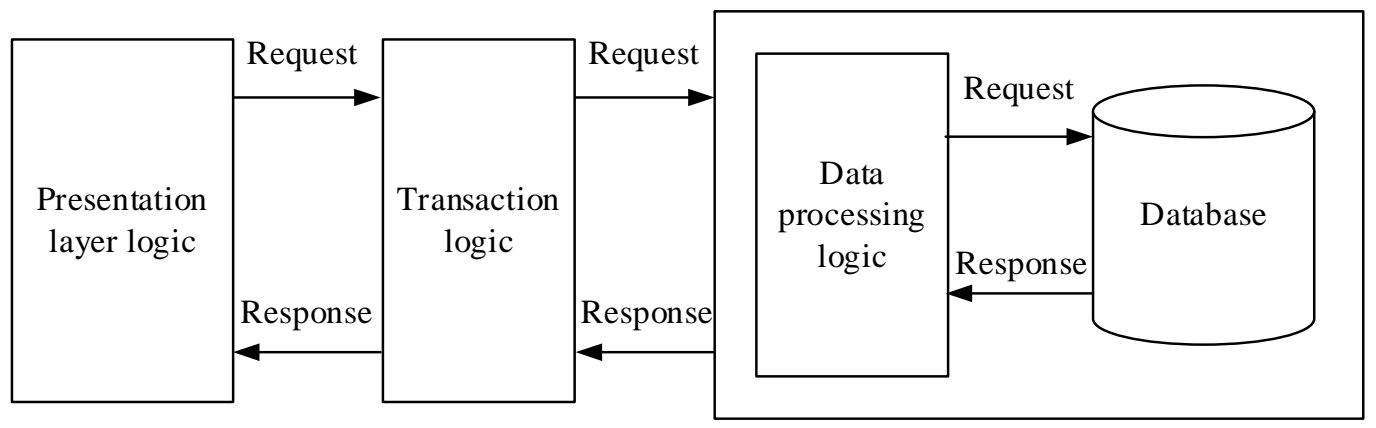

Figure 1. Architecture of B/S Mode

Presentation layer. That is a Web browser, its task is responsible for obtaining user input data, complete the data entry validation, service requests to a Web server on the network from the Web browser, Web server user identity After verifying with the HTTP protocol client requests and data transfer operations to the business logic layer, and then the operation will be completed by the user requesting the operation results transmitted by Web protocol to the client, the client accepts came home file and displays it in a Web browser on.

Business logic layer. Including one or more components and services, they apply business rules to achieve the completion of the application logic and data processing applications needed to run. As part of this process, the intermediate layer is responsible for processing the data from the data stored or transmitted to the data store.

Data layer. That database server, is the bottom of the entire layered architecture, the layer that contains data storage and components or services to interact with it. These components and services on function and business logic independent of each other (though not necessarily physically independent of each other). Data layer is to provide services business logic layer, extract data from the database according to the requirements of the business logic layer or modify data in the database.

Such a three-tier architecture greatly reduce the pressure on the client, not the distribution of load-balanced to the Web server. Since the client part of the transaction processing logic points to the server, no longer responsible for handling mission-critical complex calculations and data access, etc., it is only responsible for the display section, so maintenance personnel for the program is no longer maintenance work back and forth between each client, and to focus on the work of the functional server update on the program. This three-layer structure are independent layers, change does not affect the function of any one of the other layers.

\section{The overall structure of industrial information monitoring system}

Through the above analysis of the industrial control network remote monitoring mode, according to the task of remote monitoring network, consider the entire system uses web technology $\mathrm{B} / \mathrm{S}$ implementation structure. Based on $\mathrm{B} / \mathrm{S}$ of industrial information monitoring network according to different industrial control network and the specific applications, in their architecture and implementation technology will vary, but in general there are on-site monitoring computer, database server, death server firewall or proxy server and remote client, the structure of the whole system is a distributed control system, field IPC complete real-time sampling, the data recorded in the database field and achieve on-site monitoring. IPC site also upload the data to the control server, so each terminal station can achieve the same monitor as the scene shown in Figure 2. 


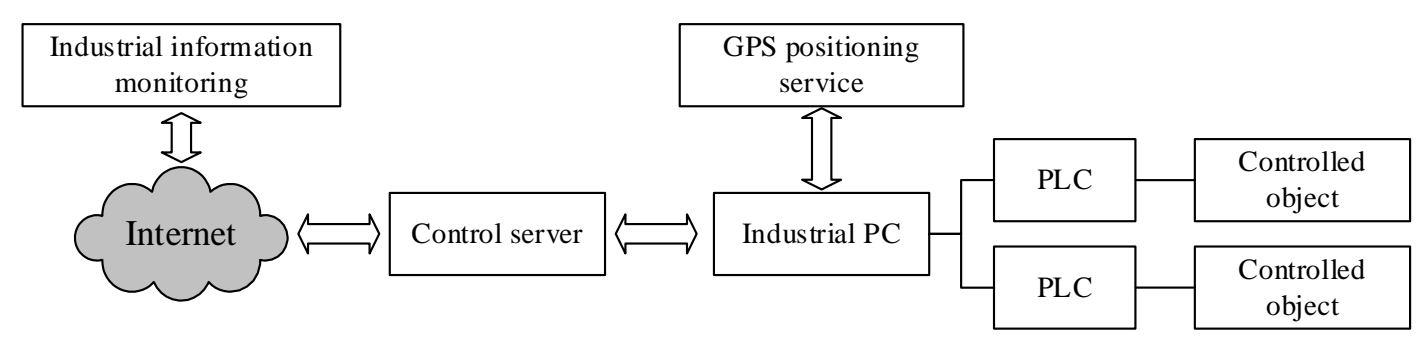

Figure 2. The overall structure of industrial information monitoring system

Site monitoring computer and the field devices exchange information to achieve regular monitoring of system functions on-site monitoring software, now with the 'field monitoring computer connected to the fieldbus monitoring and control equipment, measurement and control field work and historical data, statistical data etc. In addition it can be stored in the database as a remote monitoring agent to accept remote client commands and parameters, and these commands interpreted the results of the implementation of data required to remote client or command back to the remote client. When necessary, it also provides real-time data services. Production process historical data, real-time monitoring and control equipment field intelligent management of data, operating rights to the remote user's data, remote operation logs are all stored in the database. Web server can interact with information and services, when the remote user needs for remote operation, first log on to the website, download the appropriate page, it can be remotely operated.

Above architecture is divided by partition function is only logical, not by physical devices division. This model simplifies the client's design, the distribution system is relatively concentrated, more conducive to system maintenance, system scalability and flexibility, just to web server software modifications, it can maintain the entire system network monitoring software.

\section{The implementation of information monitoring system based on B/S}

$\mathrm{B} / \mathrm{S}$ Industrial information monitoring system based on a browser can view real-time production data, production data for a large number of complex statistical analysis, provide the basis for production planning, command and control, accident analysis. Dynamic web publishing data, data released timely, accurate and refresh speed. Remote client computer and on-site monitoring computer to establish a connection, transmission and reception of data real-time high. Customers can get through a browser page directly from the monitor real-time production data and corresponding control instructions issued. Historical production data may be retained. It provides a wealth of data access tools, such as real-time dynamic process monitoring maps, real-time curve, historical curve, data query, statistics and analysis. The system functional requirements for real-time data into the operation of historical data operation, user management, system settings, and other parts. Real-time data operations include real-time data monitoring, data display, command processing, data communications and data operations such as historical data, including user management operation history data, historical data query, historical data download and historical data printing including user login, user registration, exit the system and user management system settings including system settings, parameter setting, data print setting, data type selection. System network topology diagram shown in Figure 3.

Site monitoring equipment, mainly some of the sensors, controllers and intelligent acquisition module. Its function is to collect real-time data field device environment or accept commands issued achieve some control devices, monitoring devices threads communicate with the local monitoring host through communication modules and the fieldbus.

Monitoring host. Achieve a variety of smart devices or subsystems of monitoring, display operating parameters and operating status of each smart devices, all kinds of accidents, failures and sequence of events recording, storage access card records and video subsystem video data recording, Monitoring equipment and corresponding each measuring point, fault, alarm, alarm and other events to deal with it.

Web server for remote monitoring server is the key, is the focus of this research. The main function is to provide remote monitoring of web pages. Customer through the browser to the web 
server request after the web server process, to the real-time data or historical database server, query results sent back to the web server after the form is returned to the browser, and finally the data in some intuitive Information presented to the user.

The database server main storage room environment equipment running real-time and historical data, the operating rights to the remote user's data, remote operation logs, database server database management system uses a relational database.

Client based on B/S structure of the monitoring system without having to install any software, you can use the browser to browse room equipment, environmental history information stored in a variety of real-time information, simple operation, flexibility and variety of information clear and intuitive, with a friendly The man-machine interface.

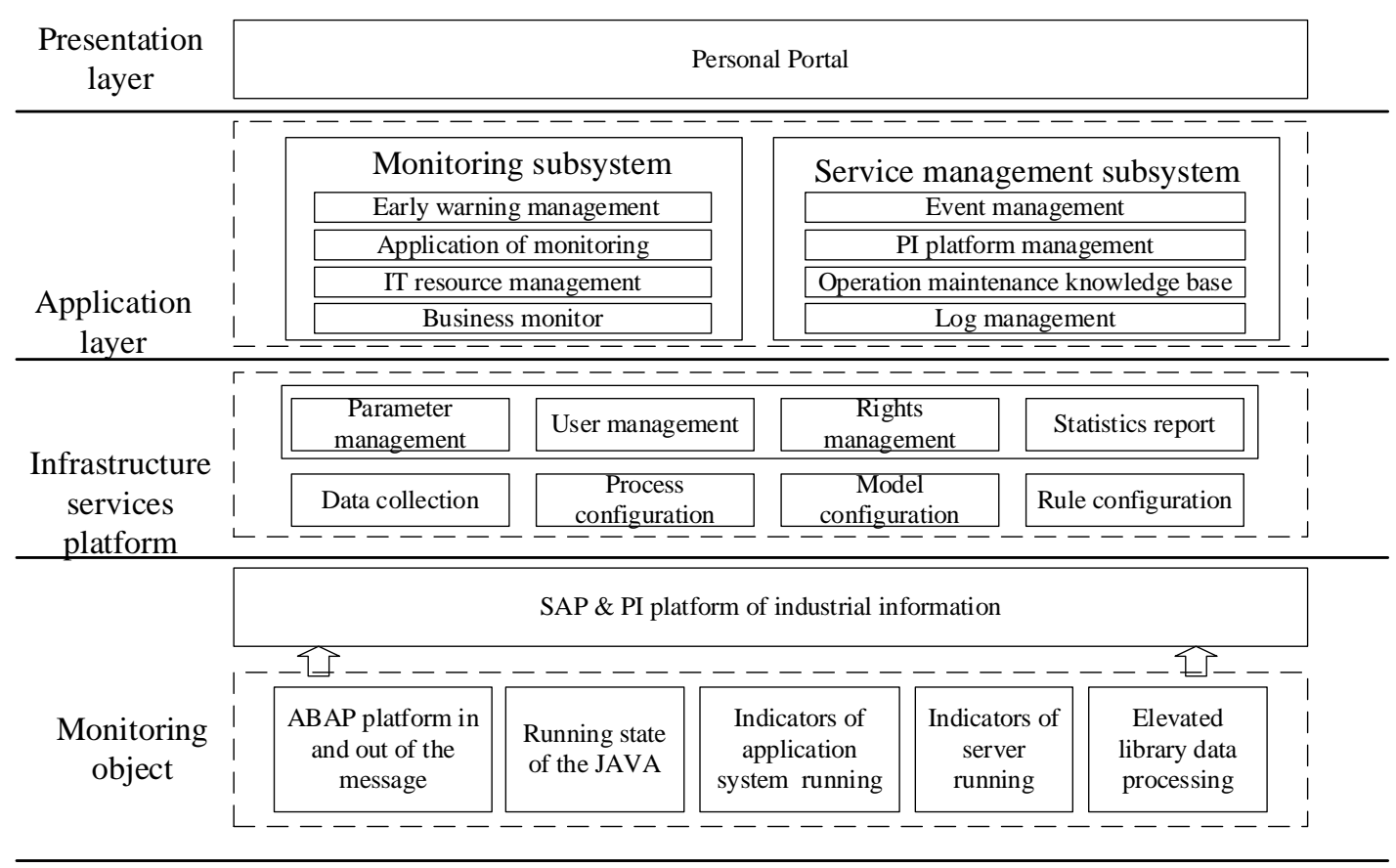

Figure 3. The implementation topology of information monitoring system based on B/S

\section{Conclusion}

Industry information monitoring enables rapid information collection, synthesis, processing, improve management decision-making for remote or unattended fewer people to achieve the purpose of downsizing for efficiency centralized management and high-level monitoring. In the rapid development of network technology, network infrastructure gradually improved today, by means of remote monitoring network has increasingly shown its advantages and feasibility. In this paper, based on B/S of industry information real-time monitoring system design. With the exponential scale was developed, we will have the ability to accept a variety of intelligent devices, such as industrial automation systems, agricultural automation systems, business automation systems, office automation systems, intelligent building systems, intelligent home systems. In short, the future everything will be intelligent, network-oriented development. New Internet technologies inevitably will greatly affect the development of remote monitoring system, remote monitoring system of the future will be a powerful, convenient, and omnipresent.

\section{Reference}

[1] Mu E, Kirsch L J, Butler B S. The assimilation of enterprise information system: An interpretation systems perspective[J]. Information \& Management, 2015, 52(3): 359-370.

[2] Cui W, Zhang S, Qi X, et al. Research on automatic monitoring system of medium and small size reservoirs based on internet of things[J]. Engineering Technology and Applications, 2014: 155. 
[3] Goyal D, Pabla B S. The Vibration Monitoring Methods and Signal Processing Techniques for Structural Health Monitoring: A Review[J]. Archives of Computational Methods in Engineering, 2015: 1-10.

[4] Swensen J S, Xiao Y, Ferguson B S, et al. Continuous, real-time monitoring of cocaine in undiluted blood serum via a microfluidic, electrochemical aptamer-based sensor[J]. Journal of the American Chemical Society, 2009, 131(12): 4262-4266.

[5] Niu G, Yang B S, Pecht M. Development of an optimized condition-based maintenance system by data fusion and reliability-centered maintenance[J]. Reliability Engineering \& System Safety, 2010, 95(7): 786-796.

[6] Young S, Gašić M, Keizer S, et al. The hidden information state model: A practical framework for POMDP-based spoken dialogue management[J]. Computer Speech \& Language, 2010, 24(2): 150-174.

[7] Lee M J, Han S, Jeon S H, et al. Electrical manipulation of nanofilaments in transition-metal oxides for resistance-based memory[J]. Nano letters, 2009, 9(4): 1476-1481 